\title{
A Dual Band Patch Antenna with a Pinwheel-Shaped Slots EBG Substrate
}

\author{
Xiaoyan Zhang, ${ }^{1}$ Zhaopeng Teng, ${ }^{1,2}$ Zhiqing Liu, ${ }^{2}$ and Bincheng $\mathrm{Li}^{1}$ \\ ${ }^{1}$ East China Jiaotong University, Nanchang 330013, China \\ ${ }^{2}$ Zhong Tian Broadband Technology Co., Ltd., Nantong 226000, China \\ Correspondence should be addressed to Zhaopeng Teng; tengzhaopeng123@163.com
}

Received 29 May 2015; Revised 6 August 2015; Accepted 11 August 2015

Academic Editor: Maria E. De Cos

Copyright (C) 2015 Xiaoyan Zhang et al. This is an open access article distributed under the Creative Commons Attribution License, which permits unrestricted use, distribution, and reproduction in any medium, provided the original work is properly cited.

A dual band microstrip patch antenna integrated with pinwheel-shaped electromagnetic band-gap (EBG) structures is proposed. The patch antenna consists of a pair of spiral slots on the patch and is fed by using coaxial line. Its full-wave simulation predicts dual bands from $4.43 \mathrm{GHz}$ to $4.56 \mathrm{GHz}$ and from $4.96 \mathrm{GHz}$ to $5.1 \mathrm{GHz}$ in the C-band. The designed EBG with eight pinwheelshaped slots addresses smaller frequency drift compared with the traditional square mushroom-like EBG when applied to the patch antenna. With the help of designed EBG structure, the impedance bandwidth, radiation efficiency, and gain of the patch antenna are improved significantly. The $10 \mathrm{~dB}$ impedance bandwidth is extended by $3.4 \%$ and $6.5 \%$ at the low- and high-frequency bands, respectively. The radiation efficiency is increased by $5 \%$ and $17.8 \%$, and the realized gain is enhanced by $1.87 \mathrm{~dB}$ and $1.56 \mathrm{~dB}$ at $4.57 \mathrm{GHz}$ and $5.06 \mathrm{GHz}$, respectively. The designed EBG structure may have many applications in other types of planar antennas.

\section{Introduction}

Microstrip patch antennas and arrays have gained tremendous interests in the embedded wireless modules due to their low profile, light weight, low cost, and easy fabrication and integration. However, their applications are limited by the low gain, low efficiency, and narrow bandwidth. The radiated field from the image of the antenna's electric current tends to cancel out the radiated field from the antenna current, thus defecting the impedance match $[1,2]$. The substrate modes in the patch substrate broaden the beamwidth of the patch antenna and reduce the radiation efficiency in the broadside direction [3]. Typically, high gain can be achieved by using large patch array $[4,5]$. However, the enhancement is not efficient due to the mutual coupling between array elements. The bandwidth of patch antennas can be improved by adding parasitic elements $[6,7]$ and designing appropriate impedance matching networks [810]. However, these structures are frequency-dependent and sensitive to fabrication tolerance. Recently, the EBG structure has been investigated to enhance the broadside gain [11], achieve wider bandwidth [12], and eliminate mutual coupling between the antenna elements [13, 14].

The EBG structure is a special family of high impedance surface (HIS) structures, which prohibits the wave propagation at certain frequencies and certain incidence angles $[15,16]$. The performance of antennas can be improved by using EBG substrates to suppress the substrate and surface modes. However, most of the EBG are applied for the singleband antennas [17-19].

In this paper, we propose a dual band spiral slot patch antenna with an EBG-based substrate. The proposed EBG structure has eight pinwheel-shaped slots on the metallic pads. Compared with the traditional mushroom-like EBG, the proposed EBG structure has smaller frequency drift when combined with the patch antenna. By using the proposed EBG, the bandwidth, broadside gain, and radiation efficiency can be improved dramatically. The paper is organized as follows. In Section 2, the design of the spiral slot patch antenna will be proposed. In Section 3, a high impedance EBG structure with eight pinwheel-shaped slots at its fringe of each EBG unit is designed. The patch antenna combined 


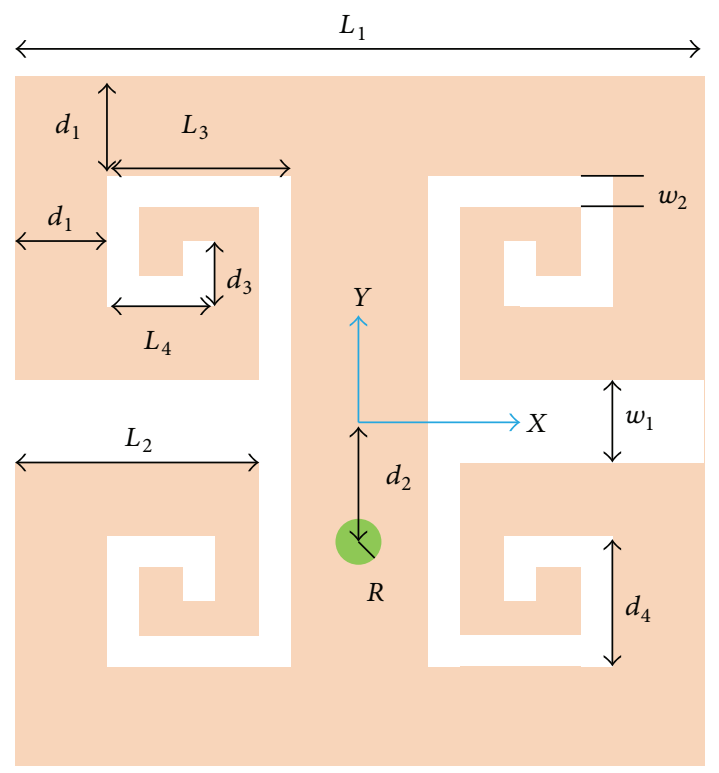

(a) Top view

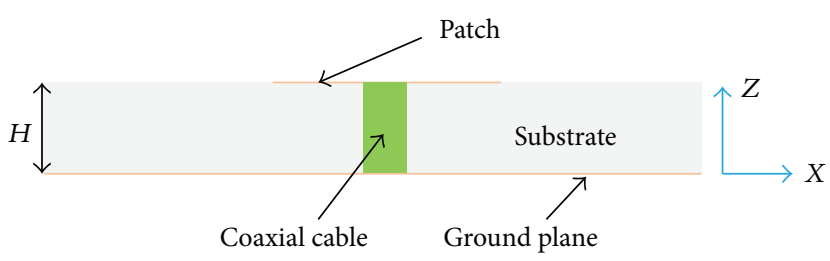

(b) Side view

FIGURE 1: Geometry of the spiral slot patch antenna.

with EBG structures is presented in Section 4. In the end, the conclusion is given in Section 5.

\section{Spiral Slot Microstrip Patch Antenna Design}

Microstrip patch antennas have been fully investigated in last century [1]. Typically, the size of the antenna is determined by the following formula:

$$
\begin{aligned}
W & =\frac{c}{2 f_{0}}\left(\frac{\varepsilon_{e}+1}{2}\right)^{-1 / 2}, \\
L & =\frac{c}{2 f_{0}-\sqrt{\varepsilon_{e}}}-2 \Delta L, \\
\frac{\Delta L}{H} & =0.421 \frac{\left(\varepsilon_{e}+0.3\right)((W / H)+0.264)}{\left(\varepsilon_{e}-0.258\right)((W / H)+0.8)},
\end{aligned}
$$

where $L, W$, and $H$ are the length, width, and height of antenna, respectively. $c$ is the speed of light in free space, $f_{0}$ is the center resonant frequency of antenna, $\varepsilon_{e}$ is the equivalent dielectric constant of substrate, and $\Delta L$ is the equivalent elongation.

Based on the aforementioned formulas, we design a spiral slot microstrip patch antenna in the C-band, as illustrated in Figure 1. The antenna is designed on a FR-4 substrate, whose thickness is $H=2 \mathrm{~mm}$ and the electrical parameters
TABle 1: Parameters of spiral slot patch antenna (unit: $\mathrm{mm}$ ).

\begin{tabular}{llllcc}
\hline$L_{1}$ & $L_{2}$ & $L_{3}$ & $L_{4}$ & $w_{1}$ & $w_{2}$ \\
\hline 26.7 & 9.5 & 7.6 & 3.8 & 2.9 & 1.1 \\
\hline$d_{1}$ & $d_{2}$ & $d_{3}$ & $d_{4}$ & $R$ & $H$ \\
\hline 2.9 & 4.6 & 3.8 & 6.7 & 0.6 & 2 \\
\hline
\end{tabular}

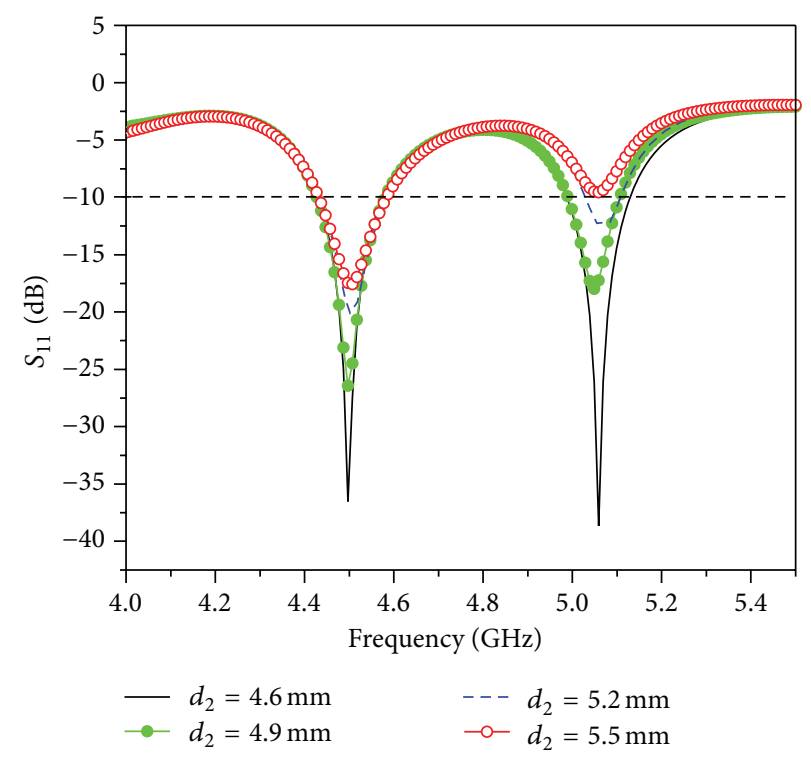

Figure 2: Simulated $S_{11}$ as a function of $d_{2}$.

are $\varepsilon_{r}=4.4$ and $\tan \delta=0.02$. The patch antenna is square and has a size of $26.7 \times 26.7 \mathrm{~mm}^{2}$. There is a pair of bilateral symmetrical T-shaped spiral slots etched on the patch surface. As Figure 1 shows, the lengths and widths of the slots are denoted by $L_{2}, L_{3}, L_{4}, d_{3}$, and $d_{4}$ and $w_{1}$ and $w_{2}$, respectively, and the distance between the slots and the patch's edge is denoted by $d_{1}$. This antenna is excited by a coaxial cable with a $50 \Omega$ SMA connector. It has a radius of $R=0.6 \mathrm{~mm}$ and is located at a distance of $d_{2}$ away from the patch center.

To investigate the effect of the feeding point's place on the return loss of the antenna, the position of the coaxial cable is tuned. In this case, $L_{1}=26.7 \mathrm{~mm}, L_{2}=9.5 \mathrm{~mm}$, $L_{3}=7.6 \mathrm{~mm}, w_{1}=2.9 \mathrm{~mm}$, and $w_{2}=1 \mathrm{~mm}$ and $d_{2}$ is varied from 4.6 to $5.5 \mathrm{~mm}$. The simulated $S_{11}$ is shown in Figure 2. The maximum return loss $\left(\left|S_{11}\right|\right)$ can be obtained at $d_{2}=4.6 \mathrm{~mm}$. At this place, the best impedance matching at $4.5 \mathrm{GHz}$ and $5.06 \mathrm{GHz}$ is observed. The optimized parameters of the antenna are listed in Table 1.

Figure 3 shows the simulated $S_{11}$ results with and without slots on the patch surface. It can be found that, after etching slots, two resonant modes generate at nearly $4.5 \mathrm{GHz}$ and $5.1 \mathrm{GHz}$, respectively. Without the slot, the patch only radiates at nearly $5.2 \mathrm{GHz}$. This is due to the fact that the excited patch surface current paths are changed by the etched slots. However, as expected, this antenna still has the drawback of very narrow bandwidth, that is, $130 \mathrm{MHz}\left(S_{11}<-10 \mathrm{~dB}\right)$ at lower frequency and $140 \mathrm{MHz}$ at higher frequency. 


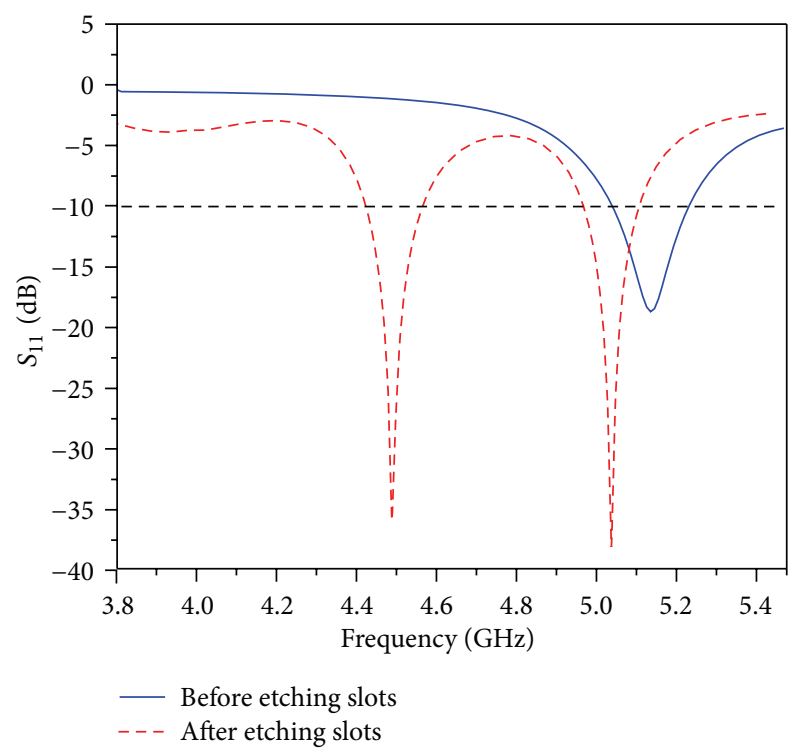

FIGURE 3: Simulated $S_{11}$ comparison before and after etching slots on the patch surface.

\section{High Impedance EBG Structure Design}

After the concept of EBG was presented, various types of EBG structures have been designed recently. The mushroom-like EBG structure has attracted more and more attention due to its small size and the distinct stop-band. The mushroom-like EBG structure consists of metallic pads, connecting vias, a bottom ground plane, and a dielectric substrate. The current flowing through the connecting via can be an equivalent inductor and the gap between the conductor edges of two adjacent cells introduces equivalent capacitance.

The resonant frequency of a $2 \mathrm{D}$ periodic LC network can be determined by the following formula:

$$
\begin{aligned}
& C=\frac{\varepsilon_{0}\left(1+\varepsilon_{r}\right) w}{\pi} \cosh ^{-1}\left(\frac{w+g}{g}\right), \\
& L=\mu h, \\
& f=\frac{1}{2 \pi \sqrt{L C}},
\end{aligned}
$$

where $w$ is the width of metallic pad, $g$ is the gap width, $h$ is the substrate thickness, $\varepsilon_{r}$ is the dielectric constant of substrate, $\varepsilon_{0}$ is the permittivity of free space, and $\mu$ is the magnetic permeability of substrate. Based on these formulas, a rough band-gap of EBG can be achieved. Then, we can get the accurate result by some slight adjustments.

There are different ways to design a periodic structure that acts as an EBG in the desired frequency range. In this paper, we design a metallic pad unit with a size of $a=7 \mathrm{~mm}$ etched eight pinwheel-shaped slots in Figure 4(a) on the basis of traditional mushroom-like EBG, and $g$ is the gap between two units. The EBG unit is at the interface of a metal-backed dielectric slab with vias connected to a ground plane. As Figure 4(a) shows, the lengths and widths of the metal pad are

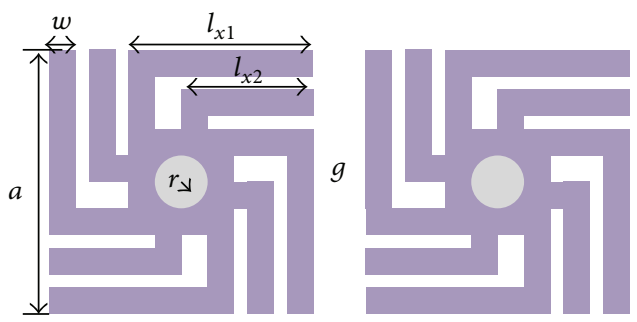

(a) Two EBG units

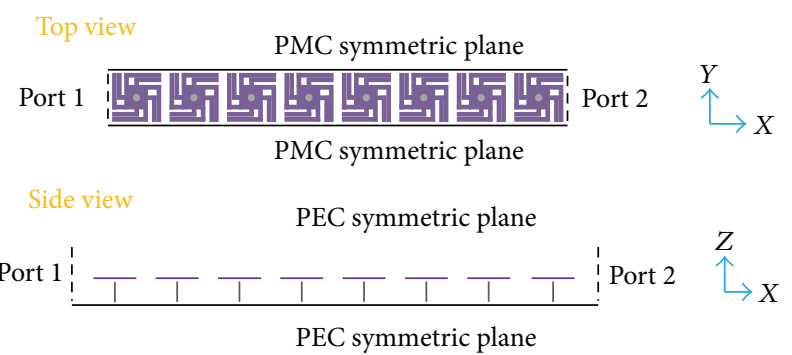

(b) Simulation model

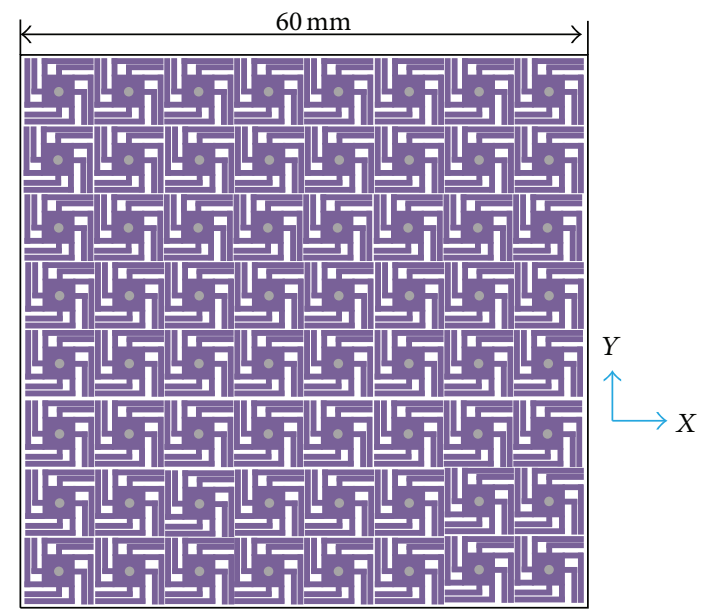

(c) $8 \times 8$ EBG structure

FIGURE 4: Geometry for the high impedance EBG structure.

denoted by $L_{x 1}, L_{x 2}$, and $w$, and the radius of the conductive via is denoted by $r$.

The technique used in the EBG simulation model is the directive transmission method [20]. We first arranged the proposed EBG cells to form a $1 \times 8$ array simulation model. Then, the simulation model is placed in ideal TEM waveguide which is a two-port waveguide formed by a pair of perfect electric conductor (PEC) along the $Z$ direction and perfect magnetic conductor (PMC) along the $Y$ direction. The input wave is launched into free space toward the inside of the waveguide at normal incidence from each port. The $1 \times 8$ array simulation model is placed in the waveguide along the $X$ direction. The detailed geometry of the simulation model is shown in the Figure 4(b). Then, it can form a TEM waveguide with this boundary condition. Finally, we can get the surface wave band-gap of finite periodic EBG structures by analyzing the transmission coefficient of the waveguide. 


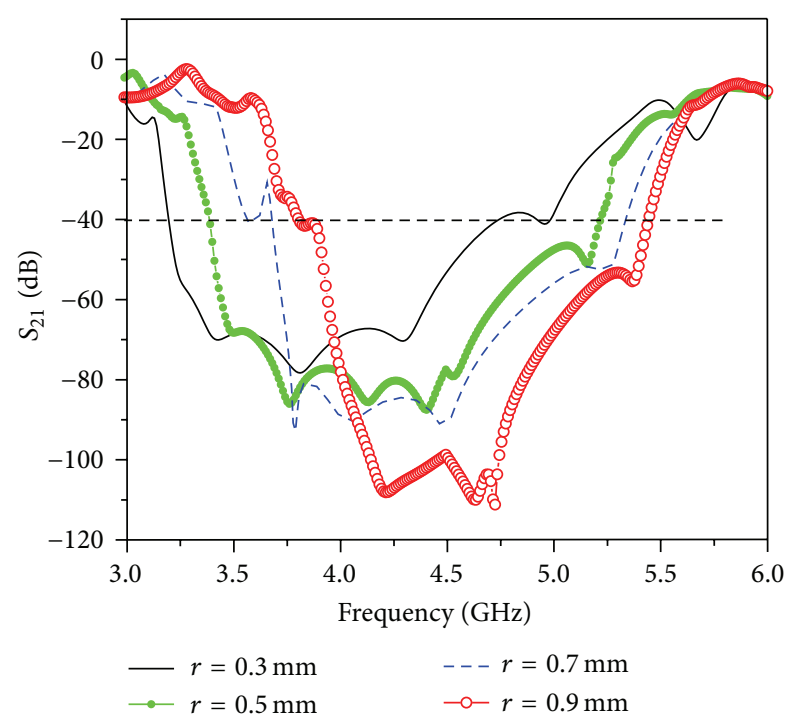

FIGURE 5: Simulated $S_{21}$ as a function of $r$.

Figure 4(c) shows the geometry of $8 \times 8$ EBG units array. As we can see, the $8 \times 8$ EBG cells are fabricated on a FR- 4 substrate $\left(\varepsilon_{r}=4.4, \tan \delta=0.02\right)$ with $2 \mathrm{~mm}$ thickness and a finite ground plane of $60 \times 60 \mathrm{~mm}^{2}$.

To optimize the EBG performance, the characteristics of insertion loss of EBG structure are studied with different parameters. A criterion of $-40 \mathrm{~dB}$ of $S_{21}$ is used to determine the band-gap of EBG structure, in which the electromagnetic wave cannot propagate due to the high surface impedance property.

Firstly, the radius of conductive via is changed. As presented in Figure 5, the band-gap of EBG structure moves to higher frequency and $S_{21}$ becomes obviously smaller when the radius gets larger. This is because the conductive via acts as equivalent inductance, according to formula, where $f$ is the resonant frequency, and $L$ and $C$ represent equivalent inductance and capacitance, respectively. Therefore, as $r$ gets larger, the equivalent $L$ gets smaller, and the resonant frequency which is the center frequency of the band-gap gets higher.

Then, the gap of the two EBG unit cells is changed from $0.3 \mathrm{~mm}$ to $0.9 \mathrm{~mm}$, as shown in Figure 6 . The wider the gap is, the higher the resonant frequency and the narrower bandwidth will be. This is attributable to the variation of the equivalent $C$ from the gap. As the gap becomes wider, the equivalent $C$ becomes smaller, so the band-gap will move to higher frequency.

The band-gap is also affected by the parameter of $w$, as is shown in Figure 7, $w$ is changed from $0.3 \mathrm{~mm}$ to $0.9 \mathrm{~mm}$, and the bigger $w$ is, the wider bandwidth will be. We also analyze how the various numbers of pinwheel-shaped slots affect the band-gap. Figure 8 shows the simulated results of $S_{21}$ with the numbers of pinwheel-shaped slots varying from 0 to 2. From this figure, we can find that the resonant frequency becomes lower with the increasing numbers of pinwheel-shaped slots. This is due to the fact that the metal pad edges are utilized more efficiently for enlarging the

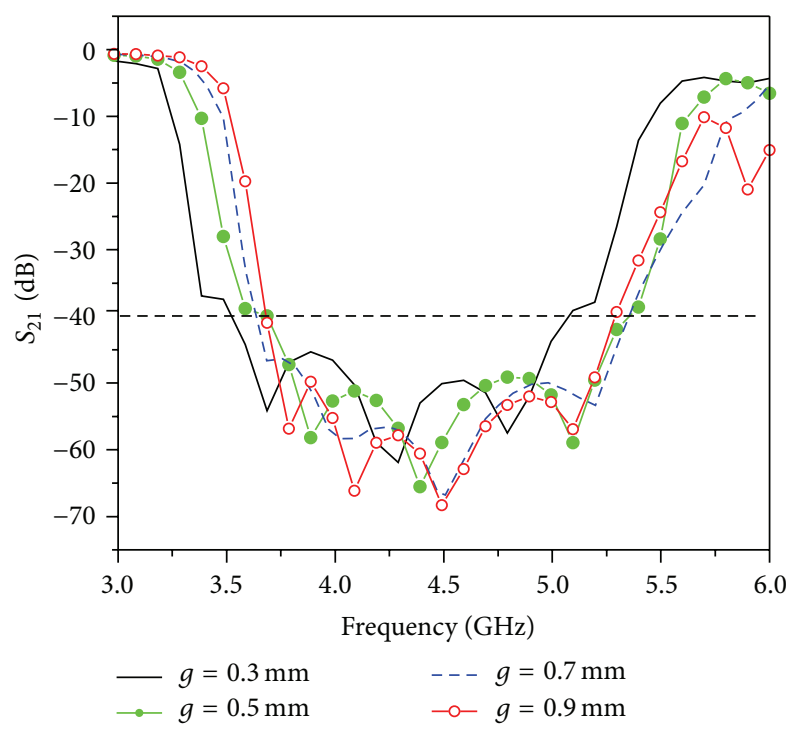

FIgURE 6: Simulated $S_{21}$ as a function of $g$.

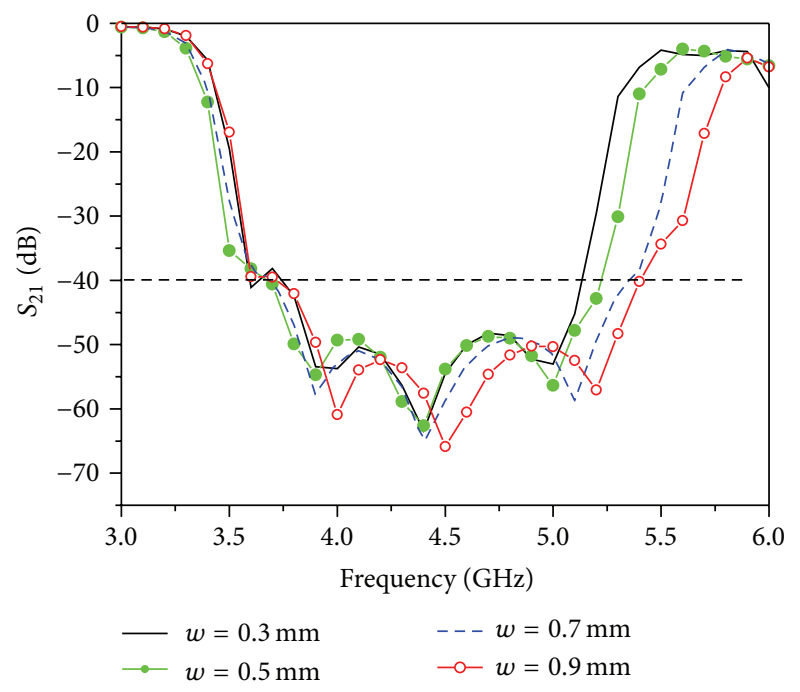

FIgURE 7: Simulated $S_{21}$ as a function of $w$.

fringe equivalent capacitance with the pinwheel-shaped slots increased [21].

According to the above analysis, the optimized parameters of the high impedance EBG structure are listed in Table 2.

\section{Spiral Slot Patch Antenna with EBG Design}

In the process of the antenna with EBG substrate design, to suppress the propagation of surface waves by using EBG structures, it is necessary to arrange some EBG units periodically. Generally, the position of the antenna also should be considered because it will influence the radiation characteristics of the antenna.

There exists the mutual coupling between the spiral slot antenna radiator and the high impedance EBG units. As we all know, the mutual coupling will change the current 
TABLE 2: Parameters of the high impedance EBG unit structure (unit: $\mathrm{mm}$ ).

\begin{tabular}{cccccc}
\hline$a$ & $g$ & $w$ & $l_{x 1}$ & $l_{x 2}$ & $r$ \\
\hline 7 & 0.5 & 0.7 & 5 & 3.6 & 0.5 \\
\hline
\end{tabular}

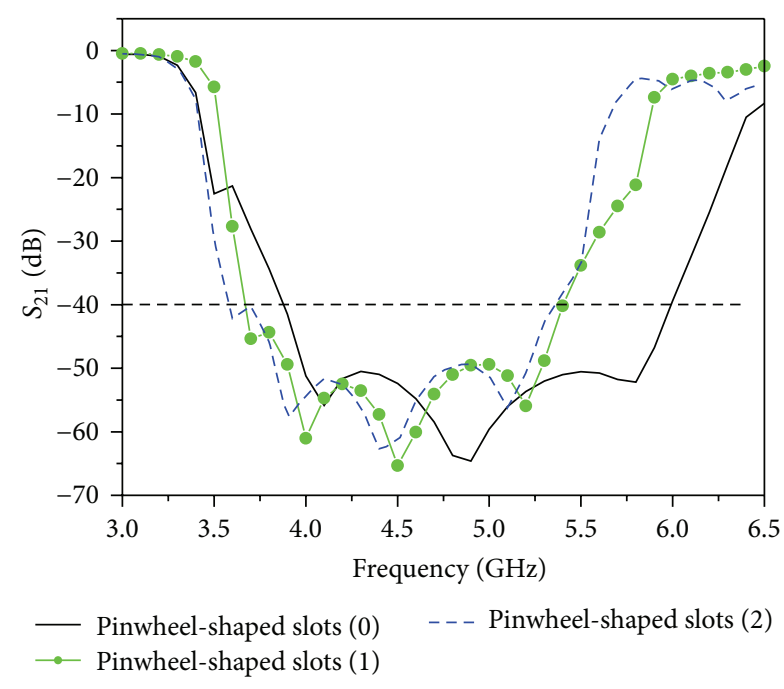

FigURE 8: Simulated $S_{21}$ as various numbers of pinwheel-shaped slots.

magnitude and phase and distribution of antenna feeds and by this way it disturbs the antenna impedance. So, the distance between antenna and EBG cells also should be taken into account.

Based on the above rules, we proposed the spiral slot patch antenna combined with EBG substrate, whose configuration of EBG antenna is illustrated in Figure 9. All the dimensions of the antenna and EBG structure are the same as in Tables 1 and 2. Two rows of EBG cells surround the slot patch antenna. The patch antenna is located in the center of a substrate, as shown in Figure 9(a). For the purpose of impedance matching, the feed location turns into $11.5 \mathrm{~mm}$ where it is measured from the patch center. The metallic pads of EBG and the spiral patch antenna are located on the same plane, that is, the XOY plane shown in Figure 9(b). An identical patch antenna with EBG substrate has also been etched on a finite substrate with the same dimension as before. A picture of the realized prototype is shown in Figure 9(c). As we can see, the metal pads of EBG and the patch antenna are placed on the same layer. So, the height of the final EBG antenna is still $2 \mathrm{~mm}$. To test the performance of the proposed EBG antenna, a coaxial cable with $50 \Omega$ SMA connector is soldered on the back side of the FR- 4 substrate.

The separation between the edge of the spiral microstrip patch antenna and edge of EBG cells, which is closest to patch antenna, is denoted by $d d$. The effect of the parameters of $d d$ on the return loss of EBG antenna is analyzed and the result is shown in Figure 10. With the value of $d d$ increasing, the impedance bandwidth of EBG antenna becomes more and more narrow. However, the frequency drift of EBG antenna becomes smaller and smaller. Considering the volume and

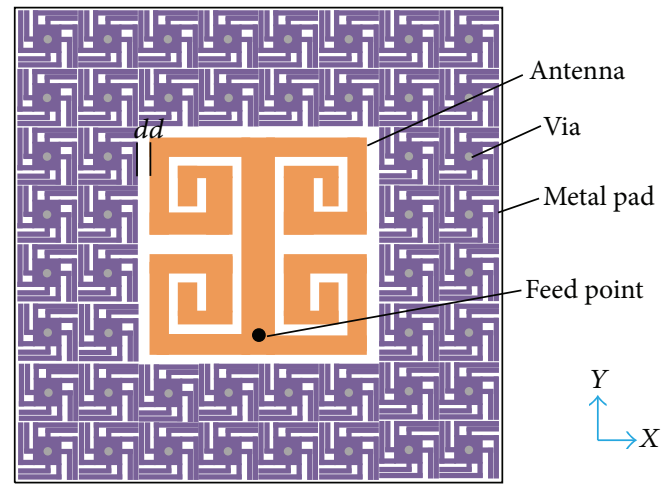

(a) Top view

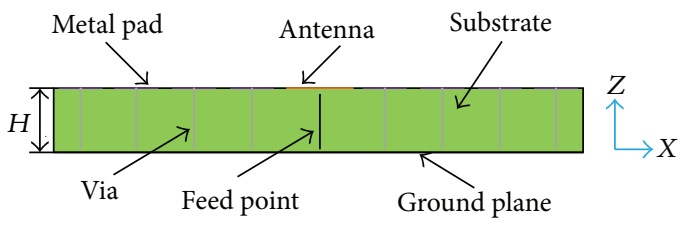

(b) Side view

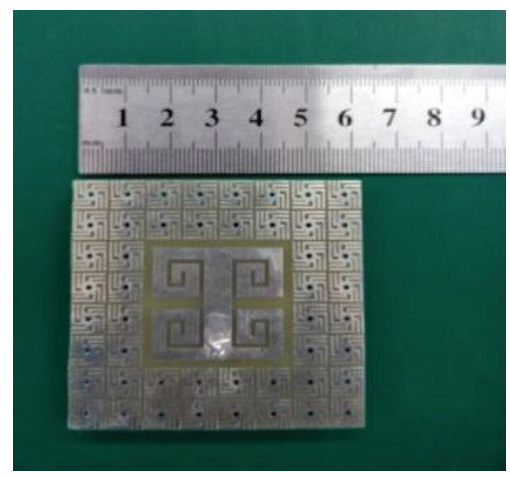

(c) Manufactured photograph

FIGURE 9: Configuration of a spiral slot patch antenna with EBG substrate.

the advanced performance of EBG antenna, the final distance is chosen as $1.9 \mathrm{~mm}$.

Figure 11 shows the simulated and measured $S_{11}$ of a single patch antenna and the proposed EBG antenna. On one hand, it can be observed that the resonant frequency of the proposed EBG antenna shifts to higher frequency slightly. Furthermore, maximum peak of the return loss of proposed EBG antenna has been degraded with respect to that of the single patch antenna. On the other hand, the single patch antenna has an input impedance bandwidth of $2.9 \%$, covering 4.43-4.56 GHz $\left(S_{11}<-10 \mathrm{~dB}\right)$, and $2.8 \%$, covering $4.96-$ $5.1 \mathrm{GHz}$. By contrast, frequency range of the proposed EBG antenna is $4.46-4.75 \mathrm{GHz}$, that is, impedance bandwidth of $6.3 \%$, and $5-5.49 \mathrm{GHz}$, that is, impedance bandwidth of $9.3 \%$. The bandwidth has been increased by $3.4 \%$ at lower frequency and $6.5 \%$ at higher frequency. For the proposed EBG antenna, the movement of resonant frequency and extension of bandwidth are all due to the coupling between the antenna elements and EBG structure. Agreement between 


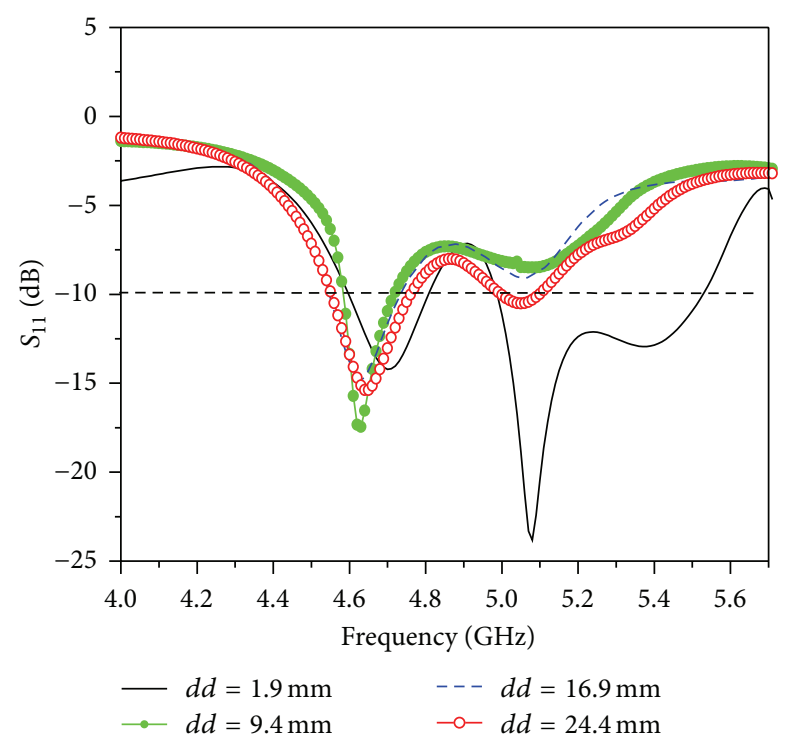

FIgURE 10: Simulated $S_{11}$ as a function of $d d$.

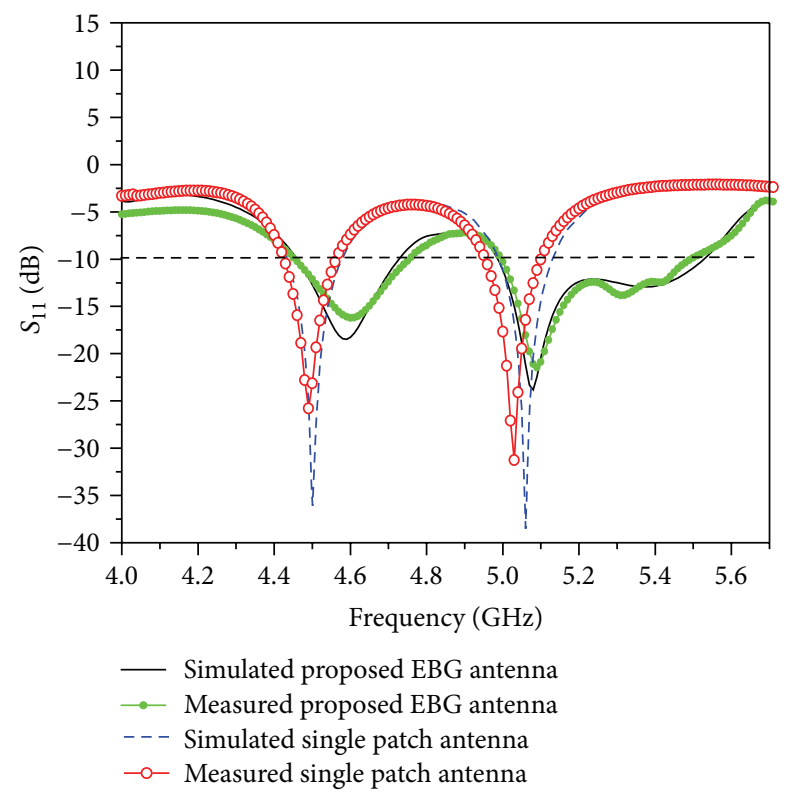

FIGURE 11: Simulated and measured $S_{11}$ of single antenna and proposed EBG antenna.

the simulation and measurement can be found from the results.

Because of the existence of the frequency shift, we should choose appropriate resonant points to compare with the radiation patterns of single antenna and proposed EBG antenna. Here, the intersections between the curves of measured proposed EBG antenna and measured single patch antenna are chosen as a criterion to calculate the radiation pattern of the antenna, that is, $4.57 \mathrm{GHz}$ and $5.06 \mathrm{GHz}$.

The measured radiation patterns of two antennas at $4.57 \mathrm{GHz}$ and $5.06 \mathrm{GHz}$ are shown in Figures 12 and 13, respectively. It can be seen from the comparison results that

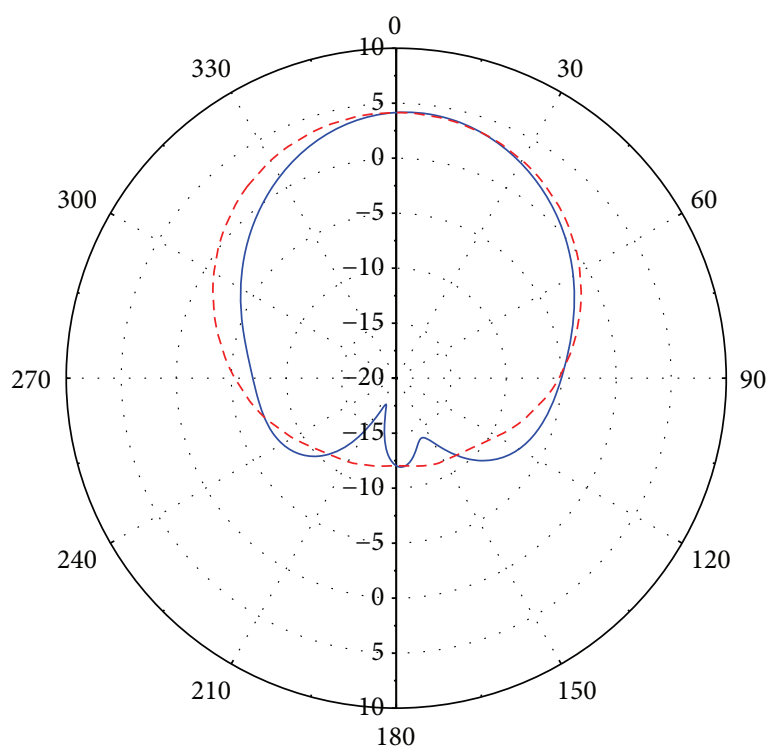

$$
\begin{aligned}
& \text { E-plane } \\
& \text {--- } H \text {-plane }
\end{aligned}
$$

(a) Single patch antenna

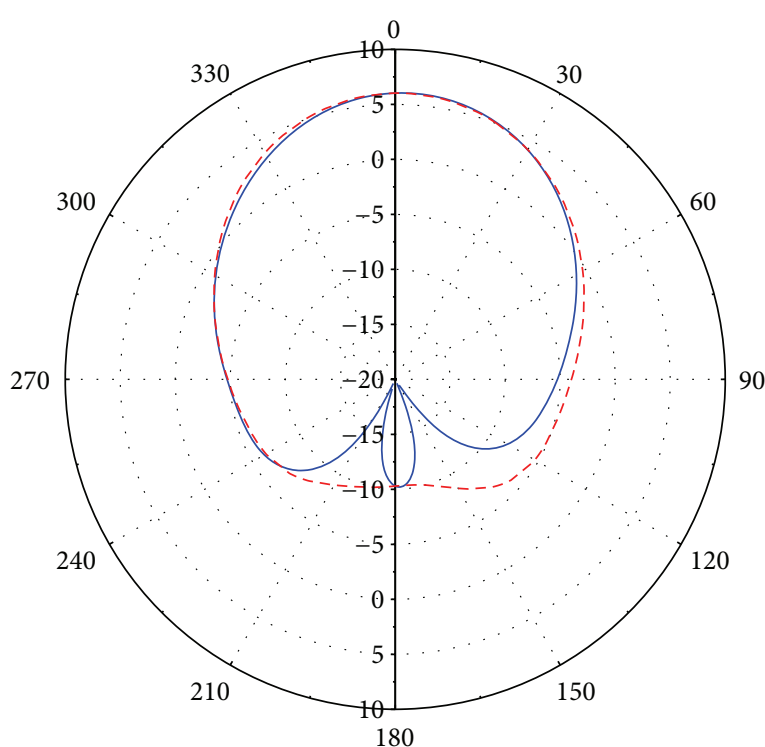

E-plane

- - $H$-plane

(b) Proposed EBG antenna

FIGURE 12: Radiation pattern of single patch antenna and proposed EBG antenna at $4.57 \mathrm{GHz}$.

the front radiation of the proposed EBG antenna is enhanced and the back radiation is also reduced compared to the single patch antenna. At $4.57 \mathrm{GHz}$, the gain of the single patch antenna is $4.15 \mathrm{~dB}$ while that of the proposed EBG antenna reaches $6.02 \mathrm{~dB}$. The radiation efficiency of the single patch antenna is $55 \%$, while that of the proposed EBG antenna is $60 \%$. The realized gain of single patch antenna increases by $1.87 \mathrm{~dB}$ after being integrated with EBG structure. At 


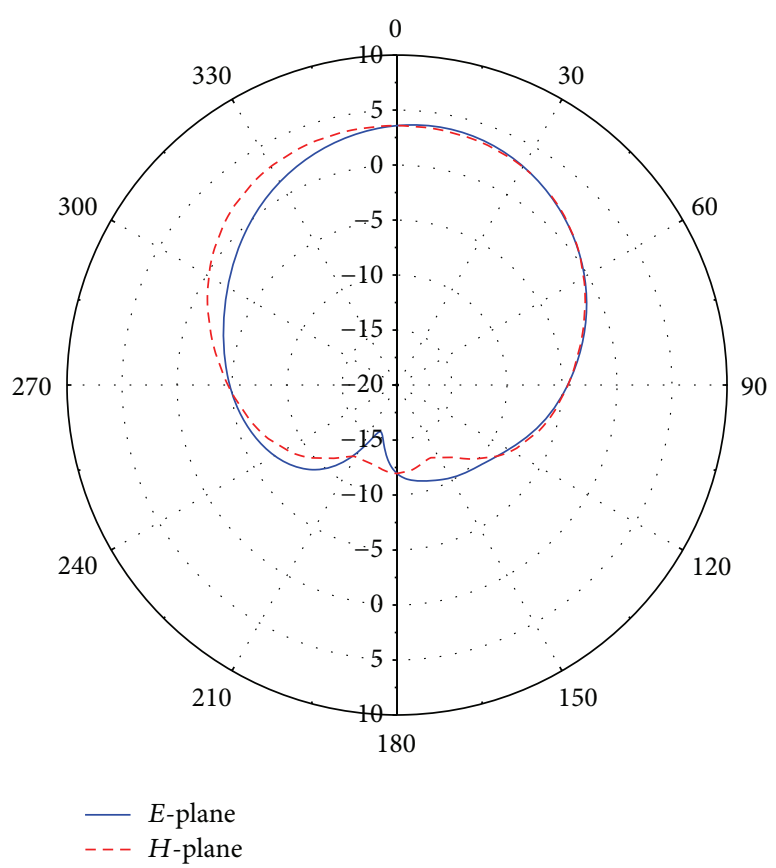

(a) Single patch antenna

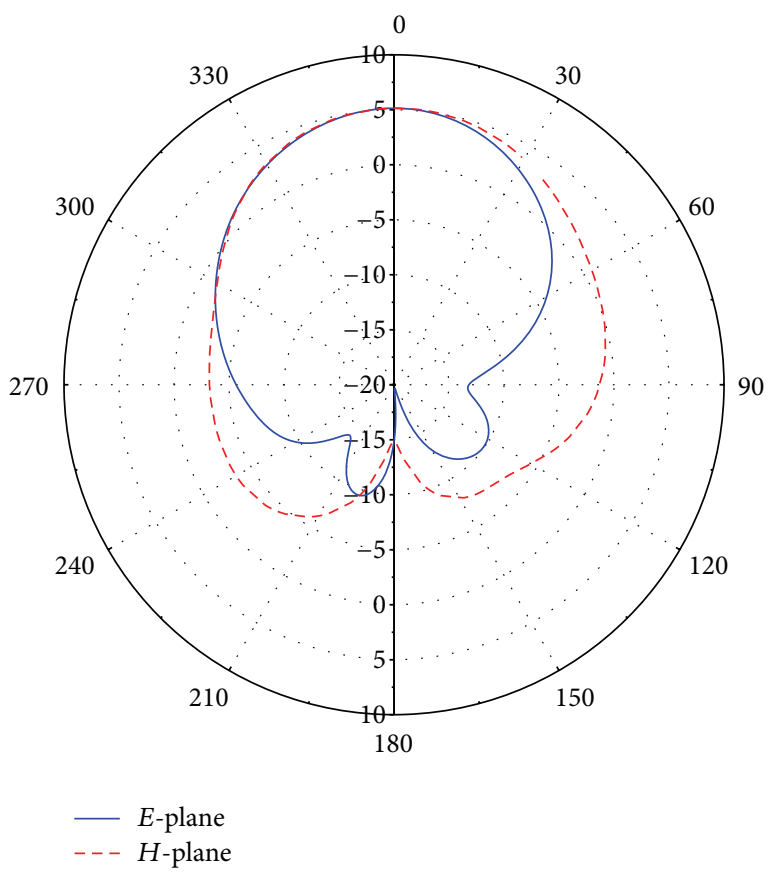

(b) Proposed EBG antenna

FIGURE 13: Radiation pattern of single patch antenna and proposed EBG antenna at $5.06 \mathrm{GHz}$.

$5.06 \mathrm{GHz}$, for the single patch antenna, realized gain is only $3.58 \mathrm{~dB}$. However, for the proposed EBG antenna, realized gain increases to $5.14 \mathrm{~dB}$. The radiation efficiencies of the single patch antenna and proposed EBG antenna are $45 \%$ and $62.8 \%$ separately. The gain and radiation efficiency of EBG antenna increase because the antenna surface wave is effectively suppressed with the help of EBG.

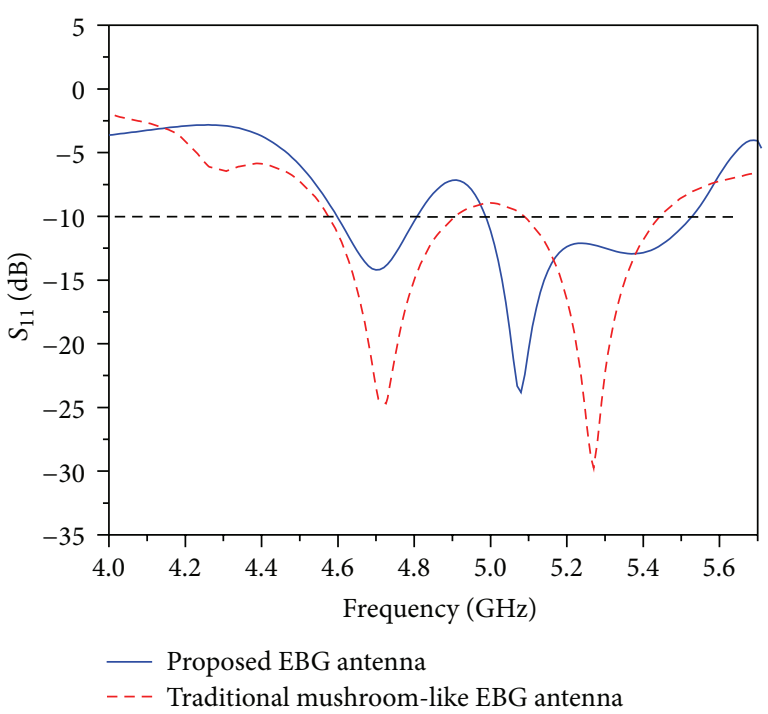

FIGURE 14: The $S_{11}$ parameters of the proposed EBG antenna and the traditional mushroom-like EBG antenna.

To present the advantage of the proposed EBG antenna further, a comparison with the patch antenna with a traditional square mushroom-like EBG substrate is analyzed. Figure 14 shows the comparison results of the parameter of $S_{11}$. As we can see, the frequency drift of our proposed EBG antenna is smaller than traditional mushroom-like EBG antenna. Compared with the traditional mushroom-like EBG antenna, the proposed EBG antenna has a wider bandwidth at higher frequency while a narrower bandwidth at lower frequency. However, the impedance matching of traditional mushroom-like EBG antenna is better than our proposed EBG antenna.

\section{Conclusion}

A spiral slot microstrip patch antenna in the C-band is presented in this paper. It has a size of $60 \times 60 \times 2 \mathrm{~mm}^{3}$. This antenna resonates at around $4.5 \mathrm{GHz}$ and $5.1 \mathrm{GHz}$, covering $4.43-4.56 \mathrm{GHz}$ and $4.96-5.1 \mathrm{GHz}$. Then, two rows of high impedance EBG cells with a band-gap of $3.6-5.5 \mathrm{GHz}$ covering the operating frequency band of antenna are designed on the substrate to surround the patch antenna. Experimental results show that the new proposed EBG antenna has wider bandwidth, which is extended to $160 \mathrm{MHz}$ at lower frequency with $60 \%$ radiation efficiency and $350 \mathrm{MHz}$ at higher frequency with $62.8 \%$ radiation efficiency, and reaches a higher peak gain, which is increased by $1.87 \mathrm{~dB}$ at $4.57 \mathrm{GHz}$ and $1.56 \mathrm{~dB}$ at $5.06 \mathrm{GHz}$, respectively. By comparing with the traditional mushroom-like EBG antenna, the frequency drift of our proposed EBG antenna is smaller. All the data show the enhanced performance of the proposed high impedance EBG antenna. 


\section{Conflict of Interests}

The authors declare that there is no conflict of interests regarding the publication of this paper.

\section{Acknowledgments}

This work was funded by China Scholarship Council. And it was supported in part by the National Natural Science Foundation (nos. 61061002 and 61261005), the 555 Talent Program of Jiangxi Province, Jiangxi Provincial Association for Science and Engineering (Jiangxi Voyage Co. [2014] no. 154), Jiangxi Natural Science Foundation (20151BAB207012), and the Science Technology Research Project of Jiangxi Education Department (GJJ14389), all in China. Special thanks are due to the Zhong Tian Broadband Technology Co., Ltd., for providing the authors with measure equipment.

\section{References}

[1] R. Garg, P. Bhartia, I. Bahl et al., Microstrip Antenna Design Handbook, Artech House, 2000.

[2] K. L. Wong, Compact and Broadband Microstrip Antennas, Wiley-Interscience, 2002.

[3] M. A. Al-Joumayly, S. M. Aguilar, N. Behdad, and S. C. Hagness, "Dual-band miniaturized patch antennas for microwave breast imaging," IEEE Antennas and Wireless Propagation Letters, vol. 9, pp. 268-271, 2010.

[4] S.-W. Su, "High-gain dual-loop antennas for MIMO access points in the 2.4/5.2/5.8 GHz bands," IEEE Transactions on Antennas and Propagation, vol. 58, no. 7, pp. 2412-2419, 2010.

[5] A. Al-Rawi, A. Hussain, J. Yang, M. Franzen, C. Orlenius, and A. A. Kishk, "A new compact wideband MIMO antennathe double-sided tapered self-grounded monopole array," IEEE Transactions on Antennas and Propagation, vol. 62, no. 6, pp. 3365-3369, 2014.

[6] R. Hosono, Y. Uemichi, X. Han, N. Guan, and Y. Nakatani, "A Bandwidth-enhanced millimeter-wave microstrip comb-line array antenna with parasitic elements on liquid crystal polymer substrate," in Proceedings of the IEEE Antennas and Propagation Society International Symposium (APSURSI '14), pp. 1726-1727, July 2014.

[7] H. Ohmine, Y. Sunahara, and M. Matsunaga, "An annular-ring microstrip antenna fed by a co-planar feed circuit for mobile satellite communication use," IEEE Transactions on Antennas and Propagation, vol. 45, no. 6, pp. 1001-1008, 1997.

[8] K. Lan, S. K. Chaudhuri, and S. Safavi-Naeini, "Design and analysis of a combination antenna with rectangular dielectric resonator and inverted L-plate," IEEE Transactions on Antennas and Propagation, vol. 53, no. 1, pp. 495-501, 2005.

[9] C. Barth, K. Van Caekenberghe, and K. Sarabandi, "An embedded broadband impedance matching technique for microstrip patch antenna," in Proceedings of the IEEE Antennas and Propagation Society International Symposium, vol. 2B, pp. 214217, July 2005.

[10] W. X. Liu, Y. Z. Yin, W. L. Xu, and S. Zuo, "Compact openslot antenna with bandwidth enhancement," IEEE Antennas and Wireless Propagation Letters, vol. 10, pp. 850-853, 2011.

[11] R. Coccioli, F.-R. Yang, K.-P. Ma, and T. Itoh, "Aperture-coupled patch antenna on UC-PBG substrate," IEEE Transactions on
Microwave Theory and Techniques, vol. 47, no. 11, pp. 2123-2130, 1999.

[12] D. Qu, L. Shafai, and A. Foroozesh, "Improving microstrip patch antenna performance using EBG substrates," IEE Proceedings: Microwaves, Antennas and Propagation, vol. 153, no. 6, pp. 558563, 2006.

[13] F. Yang and Y. Rahmat-Samii, "Microstrip antennas integrated with electromagnetic band-gap (EBG) structures: a low mutual coupling design for array applications," IEEE Transactions on Antennas and Propagation, vol. 51, no. 10, pp. 2936-2946, 2003.

[14] X. Y. Zhang, X. X. Zhong, B. C. Li, and Y. Q. Yu, "A dualpolarized MIMO antenna with EBG for 5.8GHz WLAN application," Progress in Electromagnetics Research Letters, vol. 51, pp. 15-20, 2015.

[15] E. Yablonovitch, "Photonic band-gap struc tures," Journal of the Optical Society of America B: Optical Physics, vol. 10, no. 2, pp. 283-295, 1993.

[16] D. Sievenpiper, L. Zhang, R. F. J. Broas, N. G. Alexöpolous, and E. Yablonovitch, "High-impedance electromagnetic surfaces with a forbidden frequency band," IEEE Transactions on Microwave Theory and Techniques, vol. 47, no. 11, pp. 2059-2074, 1999.

[17] F.-R. Yang, K.-P. Ma, M. Yongxi Qian, and T. Itoh, "A uniplanar compact photonic-bandgap (UC-PBG) structure and its applications for microwave circuits," IEEE Transactions on Microwave Theory and Techniques, vol. 47, no. 8, pp. 1509-1514, 1999.

[18] R. Gonzalo, P. De Maagt, and M. Sorolla, "Enhanced patchantenna performance by suppressing surface waves using photonic-bandgap substrates," IEEE Transactions on Microwave Theory and Techniques, vol. 47, no. 11, pp. 2131-2138, 1999.

[19] G.-H. Zhang, Y.-Q. Fu, C. Zhu, D.-B. Yan, and N.-C. Yuan, "A circular waveguide antenna using high-impedance ground plane," IEEE Antennas and Wireless Propagation Letters, vol. 2, pp. 86-88, 2003.

[20] L. Liang, C. H. Liang, L. Chen, and Z. J. Su, "A fast analysis method of electro- magnetic bandgap structure," High Power Laser and Particle Beams, vol. 20, no. 5, pp. 793-796, 2008.

[21] B.-Q. Lin, Q.-R. Zheng, and N.-C. Yuan, "A novel planar PBG structure for size reduction," IEEE Microwave and Wireless Components Letters, vol. 16, no. 5, pp. 269-271, 2006. 

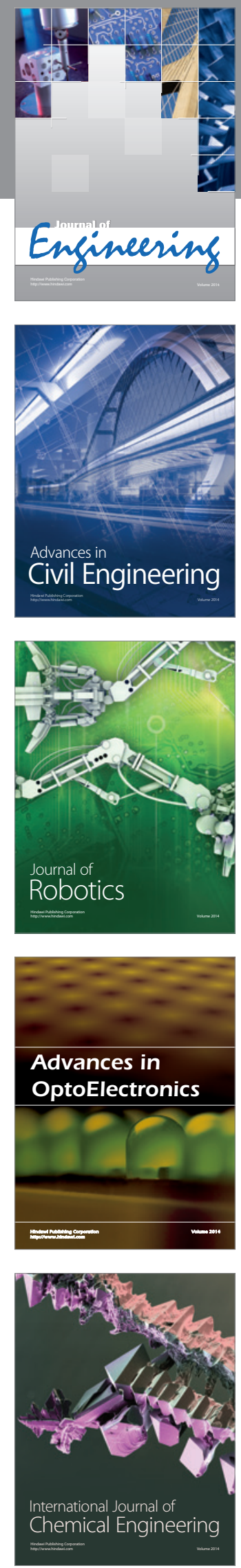

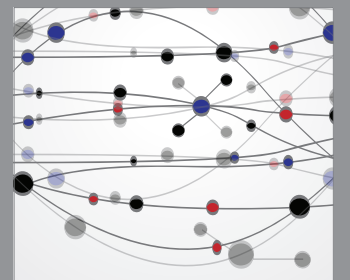

The Scientific World Journal
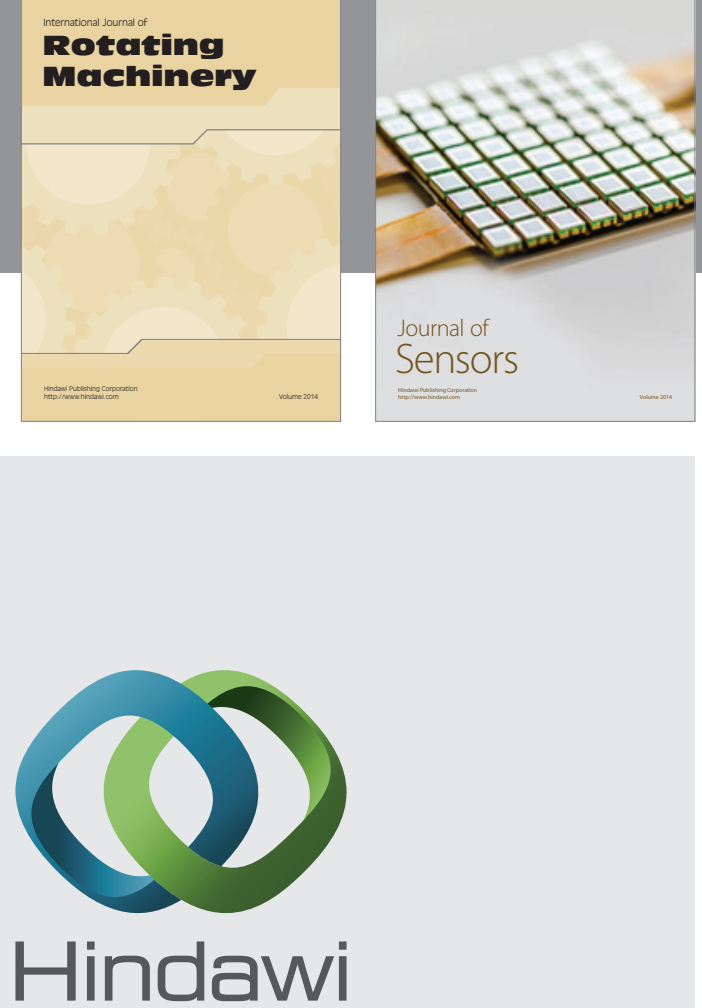

Submit your manuscripts at http://www.hindawi.com
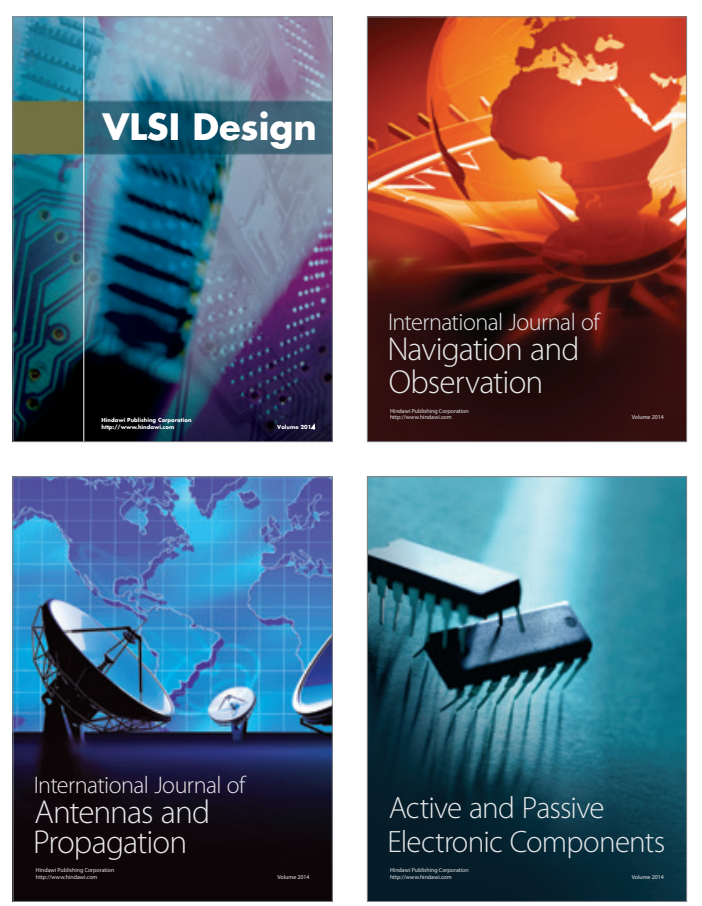
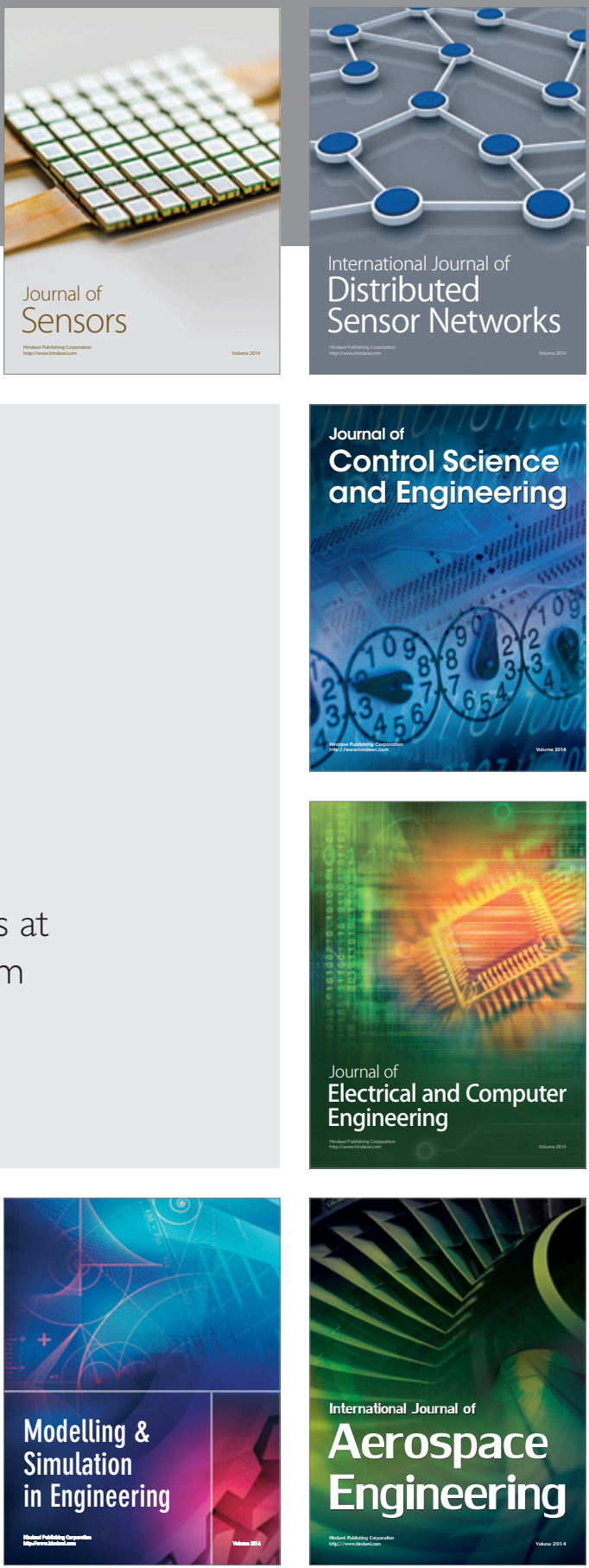

Journal of

Control Science

and Engineering
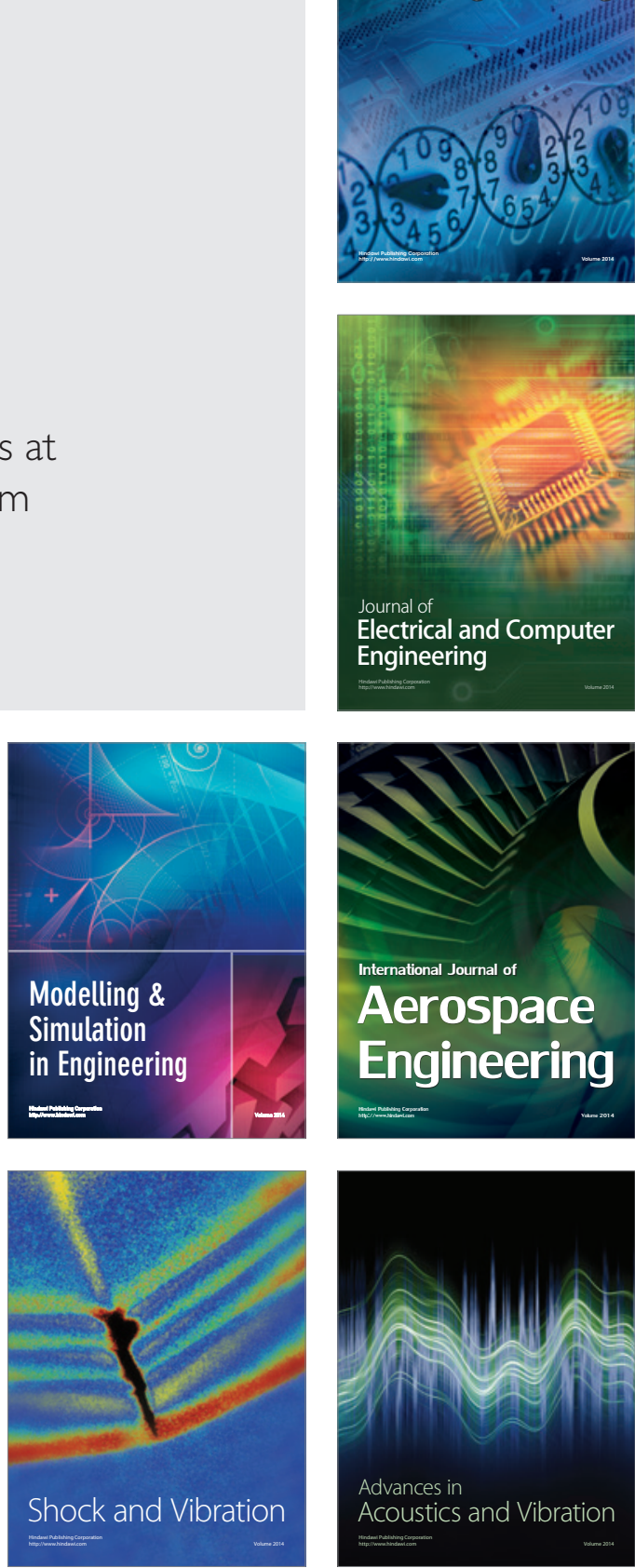